\title{
Bouveret syndrome: current management strategies
}

This article was published in the following Dove Press journal:

Clinical and Experimental Gastroenterology

\author{
Kelly M Caldwell' \\ Seeyuen J Lee' \\ Phillip L Leggett ${ }^{1}$ \\ Kulvinder S Bajwa' \\ Sheilendra S Mehta' \\ Shinil K Shah ${ }^{1,2}$ \\ 'Department of Surgery, McGovern \\ Medical School, University of \\ Texas Health Science Center \\ at Houston, Houston, TX, USA; \\ ${ }^{2}$ Michael E. Debakey Institute for \\ Comparative Cardiovascular Science \\ and Biomedical Devices, Texas A\&M \\ University, College Station, TX, USA
}

Correspondence: Shinil K Shah

Department of Surgery, McGovern Medical School, University of Texas

Health Science Center at Houston, 643I

Fannin Street, MSB 4.156, Houston, TX

77030, USA

Tel +I 7I350072I6

Email shinil.k.shah@uth.tmc.edu

\begin{abstract}
Bouveret syndrome is a rare complication of cholelithiasis that usually presents with signs and symptoms of gastric outlet obstruction. Given the relative rarity of this condition, there are no standardized guidelines for the management of this condition. In this paper, we review the diagnosis and management options (endoscopic, laparoscopic, and open approaches) of patients with Bouveret syndrome, including a report of one case to illustrate some of the endoscopic and surgical principles of management.
\end{abstract}

Keywords: Bouveret syndrome, gallstone ileus, obstruction, endoscopy, surgery

\section{Introduction}

Complications associated with cholelithiasis are common and include acute cholecystitis, choledocolithiasis, pancreatitis, and gallstone ileus. Bouveret syndrome is a rare form of gallstone ileus secondary to an acquired fistula between the gallbladder and either the duodenum or stomach. Through the fistula, a gallstone may enter the enteric system and cause a gastric outlet obstruction. Gallstone ileus is extremely rare, complicating only $0.3 \%-0.5 \%^{1}$ of patients with cholelithiasis. Bouveret syndrome represents $\sim 1 \%-3 \%$ of cases of gallstone ileus. ${ }^{1}$ Due to the rare nature of this disease, there are no standardized recommendations for the diagnostic workup and management of these patients, including endoscopic, laparoscopic, and open surgical options. In this paper, the authors review the diagnosis, workup, and management of patients with Bouveret syndrome. To illustrate pertinent points of surgical and endoscopic management, we begin with a description of a patient who was recently managed by our group.

\section{Case}

A 71-year-old man with a past medical history of atrial fibrillation, hypertension, type II diabetes mellitus, and myocardial infarction presented to the emergency department after acute onset of bright red blood per rectum followed by melena and hematemesis. He was on apixaban and had taken an extra dose the night prior to presentation. Review of systems was positive for indigestion. On physical exam, he was afebrile, slightly hypertensive, and with a regular heart rate. His abdominal exam was significant for some tenderness to palpation in the right upper quadrant and epigastrium. On initial laboratory evaluation, he had a white cell count of $14.1 \mathrm{~K} / \mathrm{CMM}$ and a hemoglobin level of $9.7 \mathrm{~g} / \mathrm{dL}$.

He was taken for esophogastroduodenoscopy (EGD) on hospital day 1 and was found to have an ulcerated lesion with an overlying blood clot in the pre-pyloric antrum (Figure 1). There was a gastric outlet obstruction preventing entry of the endoscope 
into the duodenum. Bleeding from the ulcerated lesion was controlled with endoscopic clips. Computed tomography (CT) demonstrated findings consistent with severe acute cholecystitis with a large 5-6 cm gallstone exerting mass effect on the gastric pylorus (Figure 2). He was placed on broad spectrum antibiotics until a repeat EGD and biopsies could be performed to evaluate the pre-pyloric mass (given anticoagulation with apixiban). His white blood cell count normalized and he was no longer having significant abdominal pain.

On hospital day 5, EGD with biopsies was repeated; pathology of the antral lesion demonstrated severe chronic gastritis and ulcerative changes without signs of malignancy or Helicobacter pylori infection. An upper gastrointestinal series (barium swallow study) was completed on hospital day 8 , demonstrating a $6.1 \mathrm{~cm} \times 4.1 \mathrm{~cm}$ gallstone in the stomach with contrast extravasation into the gallbladder, suggestive of a cholecystogastric fistula resulting in gastric outlet obstruction (Figure 3).

The patient underwent laparoscopy converted to open cholecystectomy with partial gastrectomy (given inability to definitely rule out malignancy as well as gastrointestinal

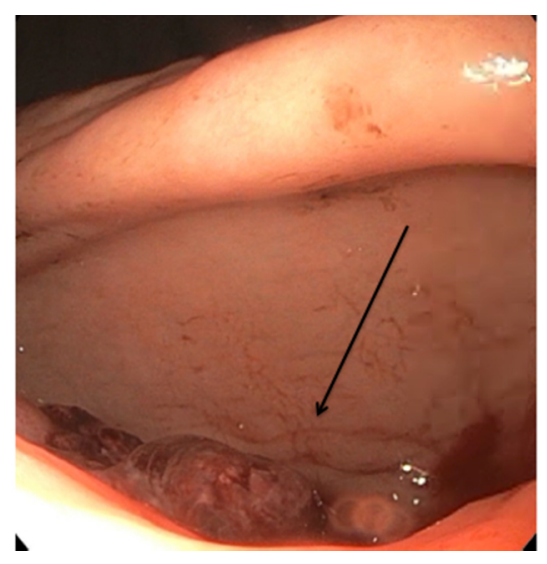

Figure I Upper endoscopy demonstrated an ulcerated lesion with an overlying blood clot in the pre-pyloric antrum (arrow).

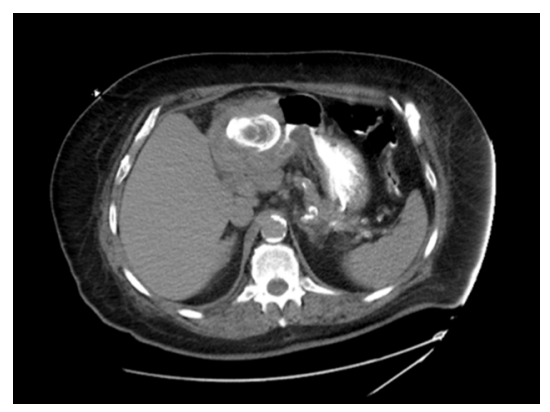

Figure 2 Initial computed tomography of the abdomen revealed significant inflammatory changes around the gallbladder with a large $5 \mathrm{~cm}$ gallstone exerting significant mass effect on the antrum of the stomach. bleed), Billroth II gastrojejunostomy, and intraoperative cholangiogram. Intraoperative findings confirmed a large gallstone in the stomach causing gastric outlet obstruction secondary to a cholecystogastric fistula. Postoperatively, the patient had delayed return of gastric function, requiring prolonged nasogastric tube as well as endoscopy. He began tolerating oral feeds on postoperative day 16 and was discharged shortly thereafter.

\section{Discussion}

\section{Diagnosis and initial management}

The first two cases of this rare condition were reported by Leon Bouveret (1896). This condition remains difficult to diagnose as the symptoms are nonspecific and the physical exam findings may be subtle. The key to diagnosing patients with Bouveret syndrome is maintaining a high level of suspicion in patients with a history of cholelithiasis and symptoms of gastric outlet obstruction. The typical patient is an older woman with multiple comorbidities, including a history of cholelithiasis, presenting with symptoms of bowel obstruction and/or gastric outlet obstruction. The largest review of 128 cases of Bouveret syndrome found that over $85 \%$ of patients diagnosed presented with nausea and vomiting. ${ }^{2}$ Abdominal pain was also seen in $\sim 70 \%$ of patients. Although less common, and similar to the case we described, patients may also present with symptoms suggesting a gastrointestinal bleed, including hematemesis (15\% of patients) and/or melena ( $6 \%$ of patients).

Most patients presenting for a suspected gastrointestinal tract obstruction are screened with an abdominal X-ray. Findings suggestive of Bouveret syndrome include Rigler's triad: a dilated stomach, pneumobilia, and a radio-opaque shadow suggesting anenteric gallstone. In patients presenting with pain in the right upper quadrant, an ultrasound may be performed. The study may demonstrate cholelithiasis with or without signs of cholecystitis, including gallbladder wall thickening or pericholecystic fluid. Upper gastrointestinal series with oral contrast may give more insight to an obstructing mass by showing a filling defect, gallstone, dilation of the stomach or duodenum, pneumobilia, and/or outlet obstruction. In rare cases, there may be contrast extravasation into the gallbladder indicating a patent cholecystoduodenal or cholecystogastric fistula (Figure 3). ${ }^{2}$

CT or MRI (Figure 4) is also commonly utilized in diagnosing these patients and may better demonstrate Rigler's triad. Oral contrast can be added which may demonstrate extravasation of contrast into the gallbladder. In the majority 


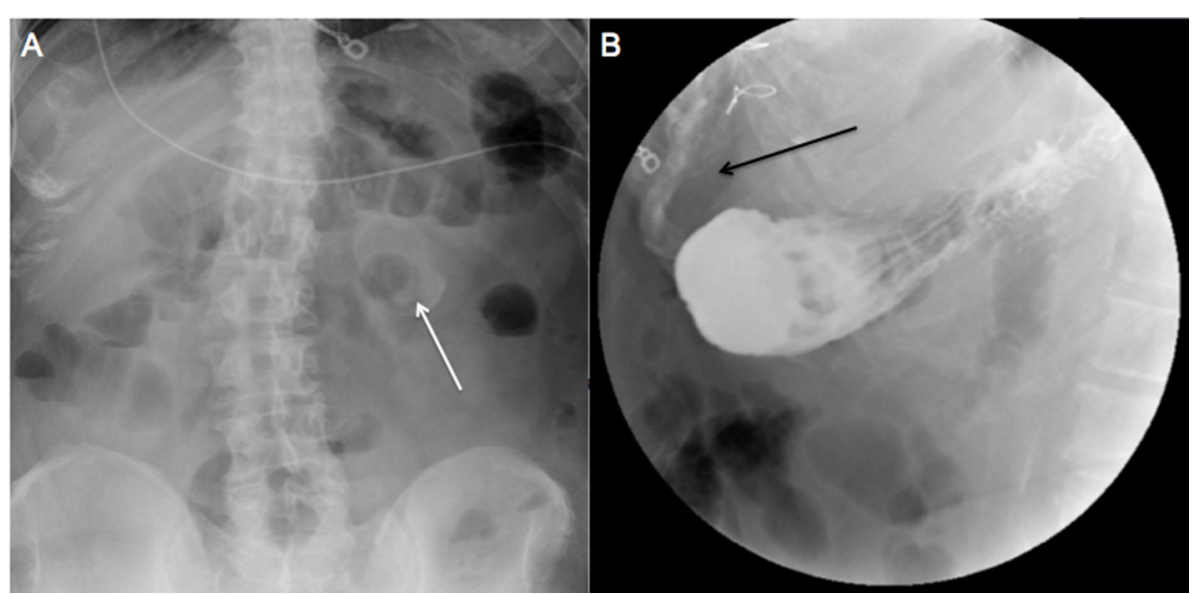

Figure 3 Initial scout films demonstrated a large 5-6 cm gallstone projecting over the left upper quadrant (white arrow; A). Upper gastrointestinal series demonstrated contrast extravasation (black arrow) into the right upper quadrant suspected to be into a contracted gallbladder and consistent with a cholecystogastric fistula (B).

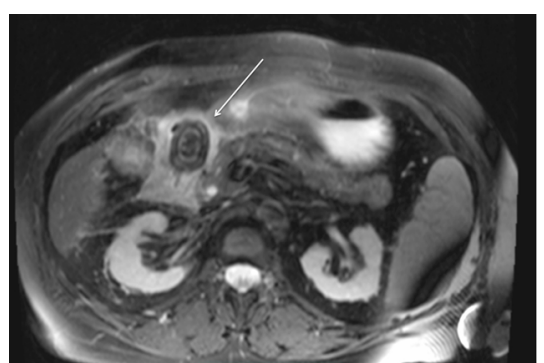

Figure $4 \mathrm{MRI}$ of the abdomen demonstrating a large impacted duodenal gallstone (arrow).

of cases, a fistula or pneumobilia will be appreciated. Gallstones may be seen in up to $50 \%$ of cases. $^{2}$

When presenting with hematemesis, patients will typically undergo EGD for diagnosis or management. As with other cases of upper gastrointestinal bleeding, active bleeding or old blood clots in the stomach or duodenum may obstruct visualization making the endoscopic diagnosis difficult. $^{3}$ In a review of clinical cases, almost all initial endoscopies demonstrated evidence of obstruction; however, visualization of the gallstone only occurred in approximately two-thirds of cases. ${ }^{2}$

Although multiple imaging modalities may be utilized in patients with Bouveret syndrome since the presenting symptoms are vague, $\mathrm{CT}$ and EGD are preferred as they are more sensitive and specific for the diagnosis of Bouveret syndrome. Treatment options for patients include a variety of endoscopic as well as surgical options and will be reviewed in the following section.

\section{Endoscopic options}

Most patients that present with Bouveret syndrome are elderly with multiple medical co-morbidities. Attempts at avoiding the morbidity and mortality of what was traditionally an open surgical approach (nearly $25 \%{ }^{4}$ ) for treatment has led to multiple reports of endoscopic therapies for this condition. As endoscopy is both diagnostic and potentially therapeutic, many are moving toward endoscopy as first-line therapy.

\section{Endoscopic removal with nets/baskets}

Multiple endoscopic modalities have been utilized for treatment of Bouveret syndrome including endoscopic nets/baskets, ${ }^{5}$ mechanical, electrohydraulic, laser, and extracorporeal shockwave lithotripsy, as well as combined endoscopic and surgical therapies. The first case of successful endoscopic treatment of Bouveret syndrome was reported by Bedogni in $1985 .{ }^{6}$ In this case, an 84-year-old woman with multiple comorbidities underwent endoscopic removal of a gallstone located in the duodenum. The stone was visualized using the endoscope and removed successfully with a basket. Since this initial report, others have reported successful removal with endoscopic nets and baskets. One case reported a simple removal of an $\sim 4 \mathrm{~cm}$ stone lodged between the first and second portion of the duodenum utilizing a Roth net. ${ }^{7}$ Basket removal of gallstones causing gastric outlet obstruction is associated with decreased surgical morbidity and mortality, is relatively simple and quick, and works well for smaller stones; however, this technique is limited as it is ineffective in removing larger stones, which typically require another endoscopic adjunct (usually some form of lithotripsy).

\section{Mechanical, electrohydraulic, laser, and extracorporeal shockwave lithotripsy}

For stones that are unable to be removed simply with an endoscopic net/basket, mechanical lithotripsy can be a use- 
ful adjunct to crush the stone prior to extraction. Typically, mechanical lithotripsy is utilized to crush the stones in the stomach or proximal duodenum, followed by endoscopic removal of stone fragments. ${ }^{8}$ Mechanical fragmentation can be accomplished by aid of a basket, snare, forceps, or a mechanical lithotripter. If malignancy is suspected, biopsies should be obtained at the time of endoscopy. It is imperative that all stone fragments be removed after mechanical fragmentation to avoid postoperative gallstone ileus complications. ${ }^{9}$

During cases that fail mechanical lithotripsy, other forms of lithotripsy may be useful; however, many of these modalities are not readily available in a general surgical operating room and often require specialized gastroenterological or urological surgery service lines. Electrohydraulic lithotripsy (EHL) is one potential option. ${ }^{4} \mathrm{EHL}$ is commonly utilized to fragment pancreatic and biliary stones. It is not as expensive as laser lithotripsy and may be more readily available in smaller hospitals. In a case reported by Sethi et al, ${ }^{40} \mathrm{a}$ double-channel therapeutic gastroscope was used for to allow for continuous irrigation. The authors report fragmenting the stone first using EHL with water immersion. The fragments were pulled into the stomach with a snare, further fragmented with a mechanical lithotripter, and then removed using a Roth net. One disadvantage to using EHL is the increased risk of damage to the surrounding tissues, including bleeding and perforation due to increased shock wave dispersion. Adequate water immersion, however, decreases the risk of these complications. Other reports of successful EHL for treatment of Bouveret syndrome have been reported. ${ }^{4}$

One of the most promising techniques for fragmenting stones is laser lithotripsy. ${ }^{10}$ Multiple techniques of laser lithotripsy have been utilized, including neodymium:YAG, ${ }^{11}$ rhodamine, ${ }^{12}$ and holmium: YAG. ${ }^{10}$ The benefit of using laser lithotripsy is precise targeting of energy onto the stone with minimal tissue injury. Some lithotripters have an additional capability of differentiating stone from tissue and interrupting signals that may potentially cause tissue damage. Some of the first successful reported cases demonstrated removal of two stones measuring 4 and $6 \mathrm{~cm}$ that were located in the duodenum. ${ }^{13}$ The gallstones were first extracted into the stomach using a basket device, and attempts were made to remove the intact stones with the basket alone. After the stones failed to pass through the esophageal hiatus, a holmium: YAG laser was utilized through the working port of the endoscope to fragment the stones. The stone fragments were then removed under direct visualization. Holmium: YAG laser lithotripsy may be advantageous over other methods given the ability to apply high energy through small, flexible probes. ${ }^{13}$ Laser lithotripsy has also been reported after failed attempts of mechanical lithotripsy. ${ }^{14}$

In addition to intracorporal techniques, successful treatment of Bouveret syndrome with extracorporeal shockwave lithotripsy (ESWL) has also been reported in the literature. In 1989, Holl et al ${ }^{41}$ demonstrated successful use of ESWL when treating an impacted duodenal gallstone with three successive treatments. Once the stone was fragmented by ESWL, the authors were able to extract the fragments via endoscopy and thus avoid surgery. Although there are cited cases of successful ESWL, most have shown to be unsuccessful or require other modes of additional therapy. When the stone is not easily visualized or accessible, ESWL may be useful in fragmenting the stone for future endoscopic removal. In a case reported by Lenz et al, ESWL was used as the impacted gallstone was too large and located in a stenotic portion of the duodenal bulb. In total, five sessions of ESWL were utilized for a 5-7 cm stone. Laser lithotripsy was required to further pulverize the stone on two separate occasions, and the fragments were removed using an endoscopic basket. ${ }^{15}$

\section{Endoscopy after open surgical treatment of Bouveret syndrome}

Endoscopy may also be considered in patients with recurrent gallstone ileus or in those who have previously undergone surgical management. Goldstein et al reported a 94-year-old patient who underwent laparotomy, enterotomy for removal of an impacted ileal gallstone as well as gastrotomy for removal of an impacted antral gallstone. Approximately 1 month postoperatively, the patient developed recurrent gastric outlet obstruction and was suspected to have recurrent Bouveret syndrome. The patient underwent endoscopy, retrieval of two impacted duodenal gallstones into the stomach, followed by laser lithotripsy (holmium:YAG) and extraction of stone fragments. ${ }^{13}$

\section{Limitations of endoscopic therapy}

It is important to keep in mind that in patients diagnosed with Bouveret syndrome, only approximately two-thirds of stones can be visualized on diagnostic EGD and even fewer can be removed successfully. Unless amenable to simple basket/net removal, endoscopic therapies typically require a high degree of expertise. Multiple modalities are sometimes required to remove the stone successfully. Consideration for placement of an esophageal overtube to protect the esophagus during stone fragment removal should be considered. ${ }^{4}$ Closure of the cholecystogastric or cholecystoduodenal fistula cannot 
be generally accomplished endoscopically; however, in the majority of cases, this is typically not necessary. ${ }^{16}$ It is imperative that all attempts be made for removal of all stone fragments after lithotripsy to avoid postoperative gallstone ileus complications. ${ }^{17}$ If the stone is inadvertently dislodged distally, one must closely follow patients for development of a more distal gallstone ileus. ${ }^{18}$

\section{Surgical options}

When endoscopy is not successful or the technical expertise is not available, surgical options may need to be considered for treatment of patients with Bouveret syndrome. One common surgical approach consists of open gastrotomy, ${ }^{19}$ pylorotomy, ${ }^{20}$ or duodenotomy ${ }^{1}$ at or immediately proximal to the site of obstruction. This is most commonly used in cases where the stone can be easily maneuvered and there is not extensive mucosal ulceration. If it is possible to maneuver an impacted duodenal gallstone into the stomach, a gastrotomy can be utilized for stone removal. ${ }^{21}$ Multiple authors have reported this approach. Open gastrotomy is also commonly used after endoscopic attempts have failed. ${ }^{22}$ In the case of a distal duodenal impacted gallstone or a gallstone that has migrated into the proximal jejunum, an enterotomy distal to the ligament of Trietz may be utilized for stone removal. ${ }^{23-27}$ The remainder of the small intestine should be examined to ensure there are no other potential large enteric stones that may result in postoperative obstruction. . $^{24,29}$

When the gallstone is located in an area that may be difficult to access during open surgery, endoscopy can be used as an adjunct to help mobilize the stone into a more favorable location to perform enterotomy. ${ }^{30}$ Thompson et al reported endoscopy utilized to retrieve impacted duodenal gallstones into the stomach, followed by surgical gastrotomy and removal of the gallstones as mechanical lithotripsy was unsuccessful to break the stones to allow for endoscopic removal. ${ }^{31}$ In a planned multi-step approach reported by Stein et al, ${ }^{30}$ a $5 \mathrm{~cm}$ gallstone located in the third portion of the duodenum was mobilized into the proximal jejunum using endoscopy with EHL and mechanical lithotripsy. Three days after endoscopy was performed, laparotomy was done to remove the stone from the jejunum. Rarely, the defect at the enterotomy site is too large for primary closure and small bowel resection or Roux-en-Y duodenojejunostomy (if stone is removed from the duodenum) is necessary for reconstruction. ${ }^{32}$

Open surgical management of Bouveret syndrome is associated with increased morbidity and mortality. If laparoscopic equipment or expertise is available, enterolithotomy under laparoscopy should be considered and has been reported as safe and effective in the management of this condition. In reported cases, successful removal of 4 and $5 \mathrm{~cm}$ stones were accomplished with good outcomes. One published report described stone removal through a duodenotomy and one described pyloroduodenotomy with transverse closure (pyloroplasty). If the stone can be manipulated into the stomach, gastrotomy can also be utilized to remove the impacted gallstone, with either suture or stapled closure of the gastrotomy. Malvaux et al described intragastric laparoscopy, in which an impacted duodenal gallstone was manipulated into the stomach, fragmented, and extracted. ${ }^{33}$ Similar to treatment with an open surgical approach, it is important after laparoscopic removal of impacted gallstones to examine the remaining small intestine to ensure there are no remaining enteric stones that may cause postoperative obstruction. In the failure of or lack of availability of endoscopic therapies, the minimally invasive laparoscopic approach to treatment should be considered as the preferred treatment in patients with Bouveret syndrome if equipment and expertise is available. ${ }^{34,35}$

Cholecystectomy with fistula repair in combination or following open or laparoscopic treatment of Bouveret syndrome remains an area of debate. Most patients are older and have multiple comorbidities making them poor surgical candidates. In these patients, the benefits of cholecystectomy generally outweigh the risk of further operations or complications from gallstones, and cholecystectomy with fistula repair is generally not recommended. However, if the fistula is not repaired, there is always a risk for a recurrent gallstone ileus and gallstone pancreatitis. ${ }^{36}$ There may an increased risk for gallbladder carcinoma in patients with cholecysto-enteric fistulas; therefore, it is recommended to perform cholecystectomy to prevent future malignant transformation in younger patients. If malignancy cannot be excluded with diagnostic imaging prior to surgery, cholecystectomy is recommended. ${ }^{37}$ Other special situations should also warrant consideration for cholecystectomy, including previous history of gallstone ileus or choledocholithiasis, gallstone pancreatitis, or concurrent hepato-pancreato-biliary malignancy amenable to surgical resection. ${ }^{38}$ There has been a report of Bouveret syndrome in a patient who had a previous partial gastrectomy and Billroth II reconstruction presenting with gallstone ileus, requiring colonoscopy to remove an impacted ascending colon gallstone, followed by endoscopy with mechanical lithotripsy to remove a gallstone impacted in the stomach, endoscopic retrograde cholangiopancreatography to remove a common bile duct stone as well as a newly found gallstone in the stomach, followed by cholecystectomy and closure of the cholecystoduodenal fistula. ${ }^{39}$ 


\section{Conclusion}

Bouveret syndrome is a rare diagnosis that affects $<0.5 \%$ of patients with gallstones. It requires an extremely high degree of suspicion for diagnosis and may be noted incidentally as part of the initial imaging workup in patients presenting with symptoms of gastric outlet obstruction. Close collaboration between therapeutic endoscopists and surgeons is important. Although there are no standardized protocols for the management of these patients, endoscopy is a useful initial diagnostic, confirmatory, and potentially therapeutic step. In the case of impacted gallstones larger than $2-3 \mathrm{~cm}$, the endoscopist should be prepared to utilize multiple endoscopic adjuncts including lithotripsy to maximize success rates. In the case of unsuccessful endoscopy or if endoscopic expertise is not available, operative treatment is generally warranted. Laparoscopic extraction of the impacted gallstone through a gastrotomy or duodenotomy should be considered the treatment of choice if the expertise is available. Cholecystectomy with treatment of the underlying fistula is generally not required in the majority of cases, but should be considered in special situations as discussed in detail in this manuscript.

\section{Acknowledgment}

The patient in this case report has provided written informed consent for the publishing of case details and accompanying images.

\section{Disclosure}

Phillip L Leggett is a consultant for Teleflex, Neosurgical, and Mauna Kea Technologies. Shinil K Shah received research grant funding from Neosurgical and Medigus. The authors report no other conflicts of interest in this work.

\section{References}

1. Qasaimeh GR, Bakkar S, Jadallah K. Bouveret's syndrome: an overlooked diagnosis. A case report and review of literature. Int Surg. 2014;99(6):819-823.

2. Cappell MS, Davis M. Characterization of Bouveret's syndrome: a comprehensive review of 128 cases. Am J Gastroenterol. 2006;101(9): 2139-2146.

3. Jones TA, Davis ME, Glantz AI. Bouveret's syndrome presenting as upper gastrointestinal hemorrhage without hematemesis. Am Surg. 2001;67(8):786-789.

4. Makker J, Muthusamy VR, Watson R, Sedarat A. Electrohydraulic lithotripsy and removal of a gallstone obstructing the duodenum: Bouveret syndrome. Gastrointest Endosc. 2015;81(4):1021-1022.

5. Zhao JC, Barrera E, Salabat M, Denham W, Leung D, Ujiki M. Endoscopic treatment for Bouveret syndrome. Surg Endosc. 2013;27(2):655.

6. Bedogni G, Contini S, Meinero M, Pedrazzoli C, Piccinini GC. Pyloroduodenal obstruction due to a biliary stone (Bouveret's syndrome) managed by endoscopic extraction. Gastrointest Endosc. 1985;31(1): 36-38.
7. Jindal A, Philips CA, Jamwal K, Sarin SK. Use of a Roth Net Platinum Universal Retriever for the endoscopic management of a large symptomatic gallstone causing Bouveret's syndrome. Endoscopy. 2016;48(S 01):E308.

8. Afzal M, Ghosh D, Leigh T. Mechanical lithotripsy for Bouveret's syndrome. Gut. 2007;56(5):733-734; author reply 734.

9. Alsolaiman MM, Reitz C, Nawras AT, Rodgers JB, Maliakkal BJ. Bouveret's syndrome complicated by distal gallstone ileus after laser lithotropsy using Holmium: YAG laser. BMC Gastroenterol. 2002;2:15.

10. Saldana Duenas C, Fernandez-Urien I, Rullan Iriarte M, Vila Costa JJ. Laser lithotripsy resolution for Bouveret syndrome. Endoscopy. 2017;49(S 01):E101-E102.

11. Maiss J, Hochberger J, Hahn EG, Lederer R, Schneider HT, Muehldorfer S. Successful laserlithotripsy in Bouveret's syndrome using a new frequency doubled doublepulse Nd:YAG laser (FREDDY). Scand J Gastroenterol. 2004;39(8):791-794.

12. Langhorst J, Schumacher B, Deselaers T, Neuhaus H. Successful endoscopic therapy of a gastric outlet obstruction due to a gallstone with intracorporeal laser lithotripsy: a case of Bouveret's syndrome. Gastrointest Endosc. 2000;51(2):209-213.

13. Goldstein EB, Savel RH, Pachter HL, Cohen J, Shamamian P. Successful treatment of Bouveret syndrome using holmium: YAG laser lithotripsy. Am Surg. 2005;71(10):882-885.

14. Chang KC, Chen WM, Wei KL. Endoscopic treatment of Bouverets syndrome in an extremely elderly patient with Holmium: YAG laser. Ann Saudi Med. 2016;36(6):436-439.

15. Lenz P, Domschke W, Domagk D. Bouveret's syndrome: unusual case with unusual therapeutic approach. Clin Gastroenterol Hepatol. 2009;7(12):e72.

16. Reisner RM, Cohen JR. Gallstone ileus: a review of 1001 reported cases. Am Surg. 1994;60(6):441-446.

17. Puri V, Lee RW, Amirlak BA, Lanspa SJ, Fitzgibbons RJ, Jr. Bouveret syndrome and gallstone ileus. Surg Laparosc Endosc Percutan Tech. 2007; 17(4):328-330.

18. Brennan GB, Rosenberg RD, Arora S. Bouveret syndrome. Radiographics. 2004;24(4):1171-1175.

19. Keller M, Epp C, Meyenberger C, Sulz MC. Unspecific abdominal symptoms and pneumobilia: a rare case of gastrointestinal obstruction. Case Rep Gastroenterol. 2014;8(2):216-220.

20. Bhattarai M, Bansal P, Patel B, Lalos A. Exploring the diagnosis and management of Bouveret's syndrome. JNMA J Nepal Med Assoc. 2016;54(201):33-35

21. Gallego Otaegui L, Sainz Lete A, Gutierrez Rios RD, et al. A rare presentation of gallstones: Bouveret s syndrome, a case report. Rev Esp Enferm Dig. 2016;108(7):434-436.

22. Mullady DK, Ahmad J. Clinical challenges and images in GI. Gallstone impacted in duodenum causing gastric outlet obstruction (Bouveret syndrome). Gastroenterology. 2007;133(4):1075, 1394.

23. Saglam F, Sivrikoz E, Alemdar A, Kamali S, Arslan U, Guven H. Bouveret syndrome: a fatal diagnostic dilemma of gastric outlet obstruction. Ulus Travma Acil Cerrahi Derg. 2015;21(2):157-159.

24. Englert ZP, Love K, Marilley MD, Bower CE. Bouveret syndrome: gallstone ileus of the duodenum. Surg Laparosc Endosc Percutan Tech. 2012;22(5):e301-303

25. Kalwaniya DS, Arya SV, Guha S, et al. A rare presentation of gastric outlet obstruction (GOO) - the Bouveret's syndrome. Ann Med Surg (Lond). 2015;4(1):67-71.

26. Mavroeidis VK, Matthioudakis DI, Economou NK, Karanikas ID. Bouveret syndrome-the rarest variant of gallstone ileus: a case report and literature review. Case Rep Surg. 2013;2013:839370.

27. MasannatYA, Caplin S, Brown T.A rare complication of a common disease: Bouveret syndrome, a case report. World J Gastroenterol. 2006;12(16): 2620-2621.

28. Shah SK, Walker PA, Fischer UM, Karanjawala BE, Khan SA. Bouveret syndrome. J Gastrointest Surg. 2013;17(9):1720-1721.

29. Moschos J, Pilpilidis I, Antonopoulos Z, et al. Complicated endoscopic management of Bouveret's syndrome. A case report and review. Rom J Gastroenterol. 2005;14(1):75-77. 
30. Stein PH, Lee C, Sejpal DV. A rock and a hard place: successful combined endoscopic and surgical treatment of Bouveret's syndrome. Clin Gastroenterol Hepatol. 2015;13(13):A25-26.

31. Thompson RJ, Gidwani A, Caddy G, McKenna E, McCallion K. Endoscopically assisted minimally invasive surgery for gallstones. Ir J Med Sci. 2009;178(1):85-87.

32. Erlandson MD, Kim AW, Richter HM, 3rd, Myers JA. Roux-en-Y duodenojejunostomy in the treatment of Bouveret syndrome. South Med J. 2009;102(9):963-965.

33. Malvaux P, Degolla R, De Saint-Hubert M, Farchakh E, Hauters P. Laparoscopic treatment of a gastric outlet obstruction caused by a gallstone (Bouveret's syndrome). Surg Endosc. 2002;16(7):1108-1109.

34. Newton RC, Loizides S, Penney N, Singh KK. Laparoscopic management of Bouveret syndrome. BMJ Case Rep. 2015;2015. pii: bcr2015209869.

35. Yang D, Wang Z, Duan ZJ, Jin S. Laparoscopic treatment of an upper gastrointestinal obstruction due to Bouveret's syndrome. World J Gastroenterol. 2013;19(40):6943-6946.
36. Jafferbhoy S, Rustum Q, Shiwani M. Bouveret's syndrome: should we remove the gall bladder? BMJ Case Rep. 2011;2011. pii: bcr0220113891.

37. Lee W, Han SS, Lee SD, et al. Bouveret's syndrome: a case report and a review of the literature. Korean J Hepatobiliary Pancreat Surg. 2012;16(2):84-87.

38. Lopes CV, Lima FK, Hartmann AA. Bouveret syndrome and pancreatic acinar cell carcinoma. Endoscopy. 2017;49(S 01):E62-E63.

39. Fejes R, Kurucsai G, Szekely A, Luka F, Altorjay A, Madacsy L. Gallstone Ileus, Bouveret's syndrome and choledocholithiasis in a patient with Billroth II gastrectomy - a case report of combined endoscopic and surgical therapy. Case Rep Gastroenterol. 2010;4(1):71-78.

40. Sethi S, Kochar R, Kothari S, Thosani N, Banerjee S. Good vibrations: successful endoscopic electrohydraulic lithotripsy for Bouveret's syndrome. Dig Dis Sci. 2015;60(8):2264-2266.

41. Holl J, Sackmann M, Hoffmann R, Schüssler P, Sauerbruch T, Jüngst D, Paumgartner G. Shock-wave therapy of gastric outlet syndrome caused by a gallstone. Gastroenterology. 1989;97(2):472-474.
Clinical and Experimental Gastroenterology

\section{Publish your work in this journal}

Clinical and Experimental Gastroenterology is an international, peerreviewed, open access, online journal publishing original research, reports, editorials, reviews and commentaries on all aspects of gastroenterology in the clinic and laboratory. This journal is included on PubMed. The manuscript management system is completely online 